\title{
A modified ant colony algorithm for the stacking sequence optimisation of a rectangular laminate
}

\author{
Wei Wang $\cdot$ Shijun Guo $\cdot$ Nan Chang $\cdot$ \\ Feng Zhao $\cdot$ Wei Yang
}

\begin{abstract}
This paper presents a modified Ant Colony Algorithm (ACA) called multi-city-layer ant colony algorithm (MCLACA). The research attention is focused on improving the computational efficiency in the stacking sequence optimisation of a laminated composite plate for maximum buckling load. A new operator, the so-called two point interchange, is introduced and proved to be effective for reducing the convergence time and enhancing the robustness in the MCLACA performance. The laminate optimisation is subject to balanced and symmetric layup with ply contiguous and strength constraints. In order to assess the MCLACA performance, a simply supported rectangular laminate plate, which was taken as numerical example in previous research using traditional ACA and genetic algorithm (GA) is chosen as a benchmark case study. Comparing with the ACA and GA results, it is shown that the presented MCLACA has better performance in terms of computational efficiency and robustness. To demonstrate the applicability of the MCLACA to a general case, an additional example of laminate optimisation has been taken with more design variables and five different boundary conditions by finite element analysis.
\end{abstract}

\footnotetext{
W. Wang $(\varangle) \cdot$ S. Guo

School of Engineering, Cranfield University, Bedford shire,

MK43 0AL, UK

e-mail: nwpuww@163.com

W. Wang $\cdot$ N. Chang $\cdot$ F. Zhao $\cdot$ W. Yang

College of Aeronautics, Northwestern Polytechnical University, Xi'an, China

W. Wang $\cdot$ N. Chang $\cdot$ F. Zhao $\cdot$ W. Yang

Chengdu Aircraft Design and Research Institute, Chengdu, China
}

Keywords Laminate optimisation - Ant colony algorithm (ACA) - Multi-city-layer ant colony algorithm (MCLACA)

\section{Introduction}

Because of the high specific stiffness and strength property and attractive potential of tailored design for weight saving, laminated composite materials are increasingly used in aircraft structures. Associated with the material usage, development of optimisation methods for composite structure design has attracted increasing attention. Taking the laminate ply orientation and ply thickness as design variables for example, composite structures can be optimised for maximum strength with minimum weight in a specified condition. Due to the manufacture constraints in practice however, the design variables are normally limited to a small set of discrete values. Optimal design of a composite structure can be formulated as a discrete combinational optimisation problem with ply thickness or ply orientation varying within a set of specific values.

Genetic algorithm (GA) is one of the most powerful tools suitable for dealing with such combinational optimisation problem with discrete nature and design variables. Goldberg and Samtani (1986) made the first investigation into a 10-bar truss optimisation by using GA. Later, Le Riche and Haftka employed GA to maximise the failure load of a fixed thickness laminate by changing its stacking sequence (Le Riche and Haftka 1993). Chen and Karunaratne used GA to optimise the stacking sequence of composite laminates (Chen and Karunaratne 2002). Many examples presented in the open literature demonstrate that GA has excellent robustness to search for global optima in composite optimisation problems (Park et al. 2001; Sciuva et al. 2003; Muc and 
Gurba 2001; Walker and Smith 2003; Kameyama and Fukunaga 2007). Despite the advantageous of GA in dealing with such optimisation problems especially with irregular objective function and large number of local minima, GA has a serious drawback of low computational efficiency. This is because GA usually requires a large number of objective function evaluations. Therefore, a lot of modified GA has been developed to improve its performance. In Kogiso et al. (1994), they presented an application of GA to the stacking sequence optimization of a composite laminate plate for maximum buckling load. In this particular study, a binary tree and a local improvement scheme based on approximate lamination parameters were introduced to improve the convergence efficiency. Le Riche and Haftka (1995) also developed a GA with improved selection of mutation and permutation operators to incorporate the physics of the problem into the GA. The presented case shows that the average cost in a genetic search is reduced by more than $50 \%$. The Ant Colony Algorithm (ACA) is one of the latest and more promising stochastic methods for optimisation. This method was inspired by the behaviour of colonies of ants when they search for food. It was introduced in early 1990s by Dorigo and Gambardella (1997), and since then it has been successfully applied to dealing with several Non-deterministic Polynomial Solvable (NP) hard combinatorial optimisation problems, such as the travelling salesman problem (TSP; Ugur and Aydin 2009). Some engineering problems such as the feeder bus network design problem (Kuan et al. 2006) and process engineering problems (Coelho and Mariani 2008) can be solved by the ACA method. In Serra and Venini (2006), general ideas of ACA were summarized and some examples regarding plane truss optimization were presented. Laminated composite structure design can also be treated as a type of combinatorial optimisation problem and solved by the ACA. Aymerich and Serra (2008) shown that the average performance and the robustness of the ACA search strategy is competitive in general case or better than the GA or Tabu Search (TS) when applied to a laminate stacking sequence optimisation problem.

In this current paper, a modified ACA, namely the Multi City-Layer Ant Colony Algorithm (MCLACA), is introduced and used to maximise the buckling load of a laminated composite plate. Taking each of the ply orientations as design variables, the stacking sequence of the laminate at a fixed thickness is optimised. In this context, the optimisation process is transformed to a type of classic combinatorial optimization problem, the so-called Multi City-Layer TSP, and solved by applying the MCLACA. To assess the efficiency of the MCLACA, an example of laminate optimisation used in previous study by employing the GA (Kogiso et al. 1994) and traditional ACA (Aymerich and
Serra 2008) is taken as a benchmark case study. The rectangular laminate plate made of 48 plies is simply supported and subjected to in-plane bi-axial compressive loading. It is optimised for maximum buckling load subject to strength constraint. In the optimisation, the number of contiguous plies of the same orientation is limited to no more than four plies to alleviate matrix cracking problem as one of the design requirements. Compared with the benchmark GA and ACA results, the presented MCLACA has shown significantly better performance in terms of computational efficiency and robustness. To demonstrate the MCLACA applicable to a general case, an additional optimisation example for a laminate with more option of ply orientations and more design variables has also been presented. Finally to validate the above laminate buckling analysis using an approximate formula based on laminate theory, finite element analysis (FEA) of the optimised laminate has been conducted.

\section{Optimisation of a laminate}

A symmetrically laminated rectangular plate of length $a$, width $b$, made of $4 \mathrm{~N}$ plies, is shown in Figs. 1 and 2. The plate is simply supported along the four edges and subjected to uniformly distributed compressive load $\lambda N_{x}$ and $\lambda N_{y}$ in the $x$ and $y$ directions, where $\lambda$ is a load factor. In this particular case, the laminate optimisation is limited to three ply orientations at $0^{\circ}, 90^{\circ}$ and $\pm 45^{\circ}$ with plies arranged in pairs denoted by $\mathrm{O}_{2}, 90_{2}$, and \pm 45 . For the laminate made of $4 \mathrm{~N}$ plies in symmetric and balanced layup, only $\mathrm{N}$ ply orientations are necessary to be taken as design variables in the optimisation.

When the applied load factor reaches a critical value $\lambda_{n}$, the plate will buckle in a mode of $m$ and $n$ half waves in the $\mathrm{x}$ and $\mathrm{y}$ directions, respectively. The critical load factor $\lambda_{n}$ can be determined in the following formula as a function of

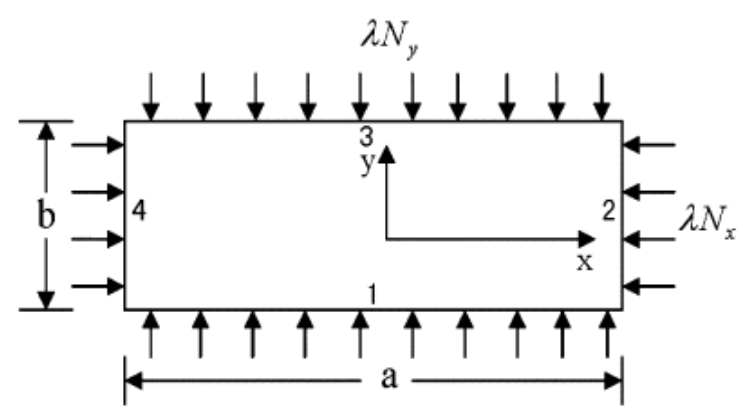

Fig. 1 Geometry of the plate and loading 


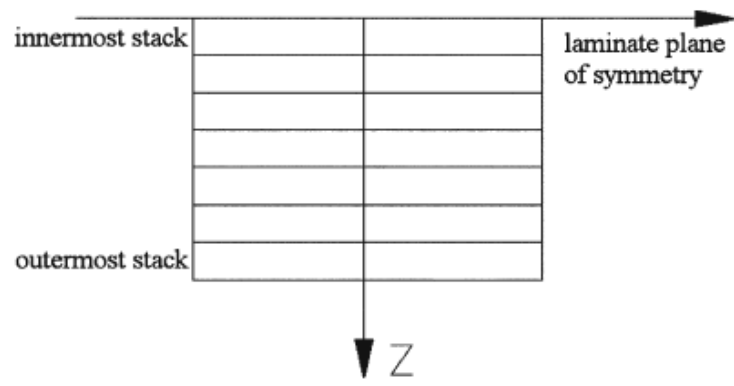

Fig. 2 Half of the 16-ply laminate through thickness

the laminate dimension, flexural stiffness $D_{i j}$ and load $N_{x}$ and $N_{y}$ (Vinson and Sierakowski 1987):

$$
\begin{aligned}
& \frac{\lambda_{n}^{(m, n)}}{\pi^{2}} \\
& =\frac{D_{11}(m / a)^{4}+2\left(D_{12}+2 D_{66}\right)(m / a)^{2}(n / b)^{2}+D_{22}(n / b)^{4}}{(m / a)^{2} N_{x}+(n / b)^{2} N_{y}}
\end{aligned}
$$

where $D_{i j}$ are the coefficients of the flexural stiffness matrix of the laminate based on the classical laminate theory. Because the laminate is designed to be symmetric and balanced, the coupling coefficients $D_{16}$ and $D_{26}$ are usually zero and the bending-torsion coupling effect can be ignored In the buckling analysis, the most concerned critical buckling load factor $\lambda_{c b}$ is determined by finding the lowest value of all combinations of the $m$ and $n$.

The strain constraint considered in the optimisation requires all strain components remain below their allowable limits. For the laminate of symmetric and balanced layup, the global shear strain $\gamma_{x y}$ is zero. According to the laminate theory, the strain components are related to the applied loads acting on the laminate by the equations:

$$
\begin{aligned}
& \lambda N_{x}=A_{11} \varepsilon_{x}+A_{12} \varepsilon_{y}, \\
& \lambda N_{y}=A_{12} \varepsilon_{x}+A_{22} \varepsilon_{y}
\end{aligned}
$$

The local strain of the ith layer in the material direction can be calculated from the global strain:

$$
\begin{aligned}
\varepsilon_{1 i} & =\cos ^{2} \theta_{i} \varepsilon_{x}+\sin ^{2} \theta_{i} \varepsilon_{y} \\
\varepsilon_{2 i} & =\sin ^{2} \theta_{i} \varepsilon_{x}+\cos ^{2} \theta_{i} \varepsilon_{y} \\
\gamma_{12 i} & =\sin 2 \theta_{i}\left(\varepsilon_{y}-\varepsilon_{x}\right)
\end{aligned}
$$

where $A_{i j}$ is the extensional stiffness matrix component of the laminate; $\theta_{i}$ is the ply orientation of the $i$ th ply. The ultimate allowable strains are $\varepsilon_{1}^{u a}=0.008, \varepsilon_{2}^{u a}=0.029$ and $\gamma_{12}^{u a}=0.015$. Taking a safety factor of 1.5 , the design allowable strains become $\varepsilon_{1}^{s a}=0.0053, \varepsilon_{2}^{s a}=0.0193$ and $\gamma_{12}^{s a}=0.010$. The critical strain failure load factor $\lambda_{c f}$ is defined as the maximum load factor $\lambda$, by which the local strain $\varepsilon_{1}, \varepsilon_{2}$ and $\gamma_{12}$ in all layers remain below the corresponding allowable strains $\varepsilon_{1}^{s a}, \varepsilon_{2}^{s a}$ and $\gamma_{12}^{s a}$.

In the optimisation problem, the objective function of the stacking sequence optimization is defined as an inverse of the more critical $\lambda_{c}$, which is the smaller one between the buckling load factor $\lambda_{c b}$ and the strain failure load factor $\lambda_{c f}$. By optimising the laminate stacking sequence, an optimisation solution can be found by searching for a minimum objective function

$\operatorname{Min} F=\frac{1}{\lambda_{c}\left(\lambda_{c b}, \lambda_{c f}\right)}$

To keep the constraint condition of symmetric and balanced layup satisfied, the laminate ply building blocks are restricted to three basic ply stacks $0_{2}, 90_{2}$ and \pm 45 in the optimisation process. Only one half of the total laminate plies are taken as design variables. In the same time, the number of contiguous plies of the same orientation limited to four is implemented in the search process of MCLACA, which will be described in the next section.

The optimisation problem of how to optimise the stacking sequence of the laminate corresponding to the objective function is described as:

Given a symmetric and balanced laminate of 48 plies, subject to in-plane compressive loads $\lambda N_{x}$ and $\lambda N_{y}$ in the $x$ and $y$ directions.

Optimize each of the laminate basic ply stacks $0_{2}, 90_{2}$ and \pm 45 to minimise the objective function $F=\frac{1}{\lambda_{c}\left(\lambda_{c b}, \lambda_{c f}\right)}$

Subject to the constraints of symmetric and balanced layup with maximum four contiguous plies of the same fibre orientation.

For this particular laminate made of 24 basic ply stacks, each ply stack has three orientation options in $0_{2}, \pm 45$ and $90_{2}$. Any option associated with a stacking sequence of the laminate can be represented in the form of a 12 bit length string. Each bit stands for one of the three orientation options. For example, if using 1, 2, 3 to represent the option $0_{2}, \pm 45,90_{2}$ respectively, the laminate of layup $\left[ \pm 45_{4} / 0_{2} / \pm 45 / 0_{4} / 90_{2} / 0_{4} / \pm 45\right]_{\mathrm{s}}$ can be represented by a string of $\left[\begin{array}{llllllllllllll}2 & 2 & 2 & 2 & 1 & 2 & 1 & 1 & 3 & 1 & 1 & 2\end{array}\right]_{\mathrm{s}}$. In the string, the rightmost number (2) corresponds to the layer closest to the laminate plane of symmetry; the leftmost number (2) represents the outermost layer. In this way, the stacking sequence optimization case may be formulated as a combinatorial optimization problem with three basic ply stacks of orientation option $\mathrm{O}_{2}, \pm 45$ and $90_{2}$. 


\section{The ant colony algorithm and modification}

The traditional ant colony algorithm (ACA) is developed based on ants' capability and behaviour in searching for the shortest route from the food source to their nest and versa. It was initially introduced by Dorigo and Gambardella (1997) in early 1990s. It is one of the latest meta-heuristic algorithms and has been successfully applied to several NP-hard combinatorial optimization problems. Travel salesman problem (TSP; Ugur and Aydin 2009) is one of the most popular optimisation problems, which attracts research attention and is usually chosen as a test-case for new optimization methods. It is one of the well-known NP-hard problems, which is difficult to be solved by traditional optimization methods. On the other hand, it is noted that the TSP is widely used in engineering applications since a typical industrial problem such as machine scheduling or cellular manufacturing can be easily modelled as a TSP. In this section, we introduce a modified version of TSP called multi city-layer travel salesman problem (MCLTSP) followed by presenting its application to modelling the laminate stacking sequence problem. Then a corresponding multi city-layer ant colony algorithm (MCLACA) is introduced to solve the MCLTSP (Fig. 3).

\subsection{Multi city-layer travel salesman problem}

The traditional TSP can be descried as finding the best order for a salesman to travel through $\mathrm{N}$ cities in a shortest route length. One city must be visited only once, and the salesman must return to the starting city at the end. In other words, the route must be a closed loop without any intersects.

In the MCLTSP, all the cities are constituted by a citymatrix. When employing the MCLTSP to describe the laminate optimisation problem represented by a 12 bit length string, a city-matrix consisting of 36 cities can be arranged in 12 columns and three lines as shown in Fig. 3. The three cities in each column represent the three optional basic ply stacks numbered in 1, 2, 3 and the 12 city layers correspond to 12 basic ply stacks. The salesman must start a tour from one of the cities in the first city-layer and visit only one city when travelling through each of the following citylayers until the last one. Unlike the traditional TSP, not all the cities but all the city-layers must be visited once in the MCLTSP. In addition, the travelling route in the MCLTSP is open. When the last city-layer (the 12th city-layer in Fig. 3) has been visited, the tour is completed and the path can be tracked by the city number being visited. For example, the path illustrated in Fig. 3 can be tracked by $\left[\begin{array}{llllllll}2 & 1 & 3 & 2 & 1 & 2 & 3 & 1\end{array}\right.$ $\left.\begin{array}{llll}2 & 1 & 3 & 2\end{array}\right]$, which represents the corresponding laminate layup $\left[ \pm 45 / 0_{2} / 90_{2} / \pm 45 / 0_{2} / \pm 45 / 90_{2} / 0_{2} \pm 45 / 0_{2} / 90_{2} / \pm 45\right]$. The route length represents the objective function described by (4) in the laminate optimisation problem. In this way, the composite optimal design problem described in Section 2 can be formulated as a MCLTSP and solved by the MCLACA with details presented in the next section.

\subsection{Multi-city-layer ant colony algorithm}

Based on the difference of the MCLTSP from the traditional TSP, the traditional ACA should be modified accordingly to develop the multi-city-layer ant colony algorithm (MCLACA). The details of applying the MCLACA to solving the composite optimization represented by the MCLTSP are described below.

In the beginning of the MCLACA process, an intensity of trail information is used to simulate the pheromone of ants. The intensity of trail information between city $i$ in the $(N-1)_{s t}$ city-layer and city $j$ in the $N_{s t}$ city-layer is denoted as $\tau(i, j, N-1)$, where $i, j=1,2, \ldots n$, and $n$ is the number of cities in a city-layer corresponding to the three ply orientation options in a basic ply stack. Without a priori available trail information, a fixed number $\tau_{0}$ in the first generation of search process was initiated as an intensity matrix of trail information.

Taking $m$ total number of ants to go through $m$ different routes from the first city-layer to the last one, the ants are randomly distributed to the cities of the first city-layer at the first step. The task for all ants is to find an objective city in the next city-layer. For an ant at city $i$ in the $(k-1)$ th
Fig. 3 Illustration of the multi city-layer model and laminate stacking sequence

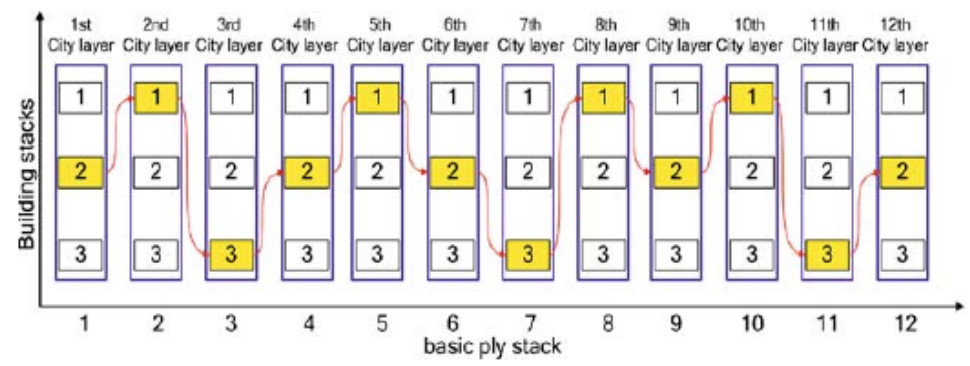


city-layer, the probability for the ant to select city $j$ in the $k$ th city-layer to visit at the next step can be written in a formula as:

$j=\left\{\begin{array}{cc}\arg \max _{u \in \text { allowed }_{k}}\left\{[\tau(i, u, k)]^{\alpha}\right\} & \text { if } q<q_{0}, \\ S & \text { otherwise, }\end{array}\right.$

where $\alpha$ represents the degree of relative importance of the trail information; $q$ is a parameter chosen randomly with uniform probability in $[0,1]$ and $q_{0}$ is a given parameter; $S$ is a random variable selected according to the following probability distribution which have a higher level of pheromone trail:

$P(i, j)=\frac{\tau^{\alpha}(i, j, k)}{\sum_{u \in \text { allowed }_{k}} \tau^{\alpha}(i, j, k)}$

The pheromone trail is updated in two levels: global updating and local updating. When each ant has completed its tour, if the edge is chosen by ant $m$, its amount of pheromone is changed by using the following local trail updating formula:

$\tau(i, j, k) \leftarrow(1-\xi) \cdot \tau(i, j, k)+\xi \cdot L(m)$

Where $\xi$ is a parameter, $L(m)$ is the route length of ant $m$.

The local trail updating is motivated by trail evaporation of real ants to avoid a high frequency selection by all the ants that could result in a local convergence and premature solution. When all the ants complete their tours in one iterative process, the route selected by each ant represents an option of stacking sequence in the laminate optimisation. In encoding the above MCLACA process to optimise the laminate stacking sequence, the route length was replaced by the objective function in terms of critical load factor shown in (4). At the end of a tour, the best ant with the shortest route length deposits pheromone on the visited edges that belong to its tour while the other edges remain unchanged. The amount of pheromone $\Delta \tau$ deposited by the best ant is proportional to the tour length. The global trail updating is a process of reinforcement learning scheme in which better solutions get a greater reinforcement expressed by a formula shown in (8):

$$
\begin{aligned}
& \tau(i, j, k) \leftarrow(1-\rho) \cdot \tau(i, j, k)+\Delta \tau \\
& \rho \in(0,1) \\
& \Delta \tau=\left\{\begin{array}{cc}
\rho / L_{g b} & \text { if }(i, j) \in \text { the longest path } \\
-\varepsilon \cdot \frac{L_{\text {worst }}}{L_{\text {best }}} & \text { if }(i, j) \in \text { the shortest path } \\
0 & \text { others }
\end{array}\right.
\end{aligned}
$$

In the process, the symmetry constrain is realized by optimizing only half of the laminate, while the balance constrain is satisfied by restricting the choice of basic building blocks to $\pm 45,0_{2}$ and $90_{2}$ ply stacks. The ply contiguity constraint is satisfied in the selection of the next city to visit by the ant. When visiting a new city, the ant checks and compares the current city against the previous two neighbour cities visited to avoid selecting the same city number already being visited twice. When applied to the laminate optimisation, this process limits the contiguous basic ply stacks up to two or the number of contiguous plies of the same fibre orientation up to four except for \pm 45 plies. For example, an ant has visited six city-layers in a rout of $\left[\begin{array}{lllllll}3 & 2 & 2 & 2 & 1 & 1\end{array}\right]$ being encoded as a laminate layup $90_{2} / \pm 45 / \pm 45 / \pm 45 / 0_{2} / 0_{2}$. Since there are four contiguous 0 plies in the layup, the next city to visit can only be selected from $\{2,3\}$.

In order to improve the convergence process of the proposed MCLACA, a two point interchange operator used to improve the GA performance in Kogiso et al. (1994) was adopted. The MCLACA performance is improved by selecting randomly and making two digits of each design string interchanged. In this manner, a one-step searching for a local optimum in a small neighbourhood of the design is added into the MCLACA process. For example, the seventh bit ' 2 ' and eighth bit ' 3 ' in the following string swaps their positions after the interchange operation.

Initial ant route: $\quad \begin{array}{llllllllllll}1 & 2 & 3 & 2 & 2 & 2 & 2 & 3 & 3 & 2 & 3 & 3\end{array}$

After interchange: $\begin{array}{llllllllllll}1 & 2 & 3 & 2 & 2 & 2 & 3 & 2 & 3 & 2 & 3 & 3\end{array}$

In the optimization process, the positions of the bits to be interchanged are chosen randomly. This may cause a violation of the ply contiguous constraint. In this case, the two point interchange is operated again from the initial ant route until the constraint is satisfied.

The procedure of the MCLACA is summarised below and illustrate in Fig. 4.

Step 1: Set parameters and initialize pheromone trails

Step 2: Allocate randomly each of the ants to a city in the first city-layer.

Step 3: Every ant must travel to a city in the next city-layer depending on the probability distribution given in (5)

Step 4: Perform a local update of pheromone according to (7)

Step 5: Calculate the route length of all paths to identify the best and worst ant, and perform a global update of pheromone according to (8)

Step 6: If the specified maximum iteration is completed, terminate the process; otherwise repeat the step $2-5$. 
Fig. 4 Basic flowchart of MCLACA

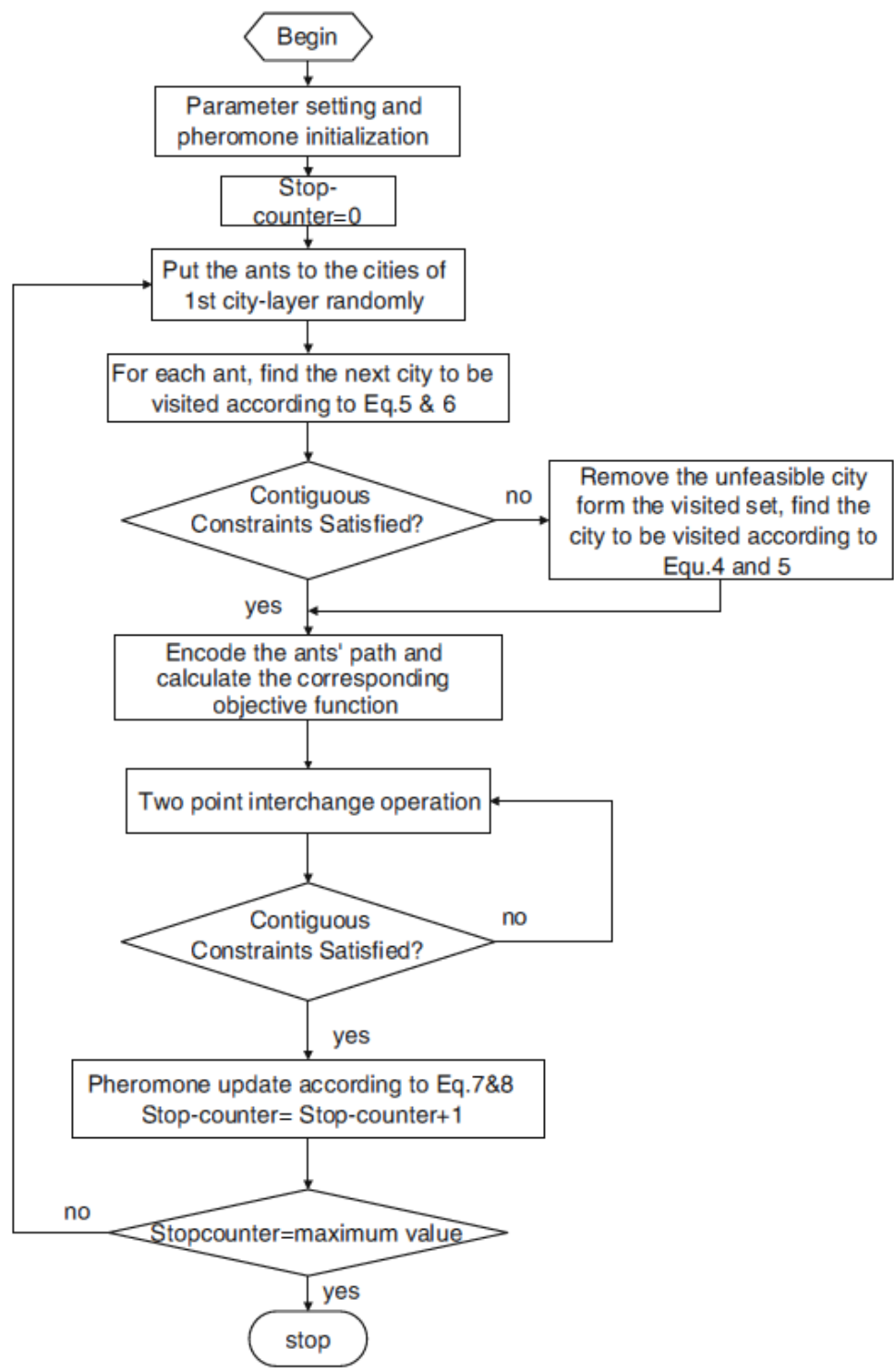

\section{Example results and discussion}

A rectangular laminate plate used in previous study by employing the GA (Kogiso et al. 1994) and traditional ACA (Aymerich and Serra 2008) is taken as a benchmark case study by using the MCLACA. The laminate plate is made of carbon/epoxy prepreg with material properties listed in Table 1.

The laminate is $\mathrm{a}=508 \mathrm{~mm}$ in length and $\mathrm{b}=127 \mathrm{~mm}$ in width, and made of $\mathrm{N}=48$ plies with ply thickness $\mathrm{t}=$ $0.127 \mathrm{~mm}$. In order to assess the computational efficiency and solution quality of the MCLACA and compare with the GA (Kogiso et al. 1994) and traditional ACA (Aymerich and
Serra 2008) results, the simply supported boundary condition and the following three load cases were considered in this example.

Load case 1: $\quad N_{x}=175 \mathrm{~N} / \mathrm{m} ; N_{y} / N_{x}=0.125$

Load case 2: $\quad N_{x}=175 \mathrm{~N} / \mathrm{m} ; N_{y} / N_{x}=0.25$

Load case 3: $N_{x}=175 \mathrm{~N} / \mathrm{m} ; N_{y} / N_{x}=0.5$

The stacking sequence of the laminate is optimised for the maximum buckling load subject to the strength constraint. As defined in Section 3, the objective function is calculated from the laminate critical load factor. To keep the laminate layup symmetric and balanced, there are only 
Table 1 Material properties of the carbon/epoxy (Kogiso et al. 1994; Serra and Venini 2006)

\begin{tabular}{lllllll}
\hline$E_{11}(\mathrm{GPa})$ & $E_{22}(\mathrm{GPa})$ & $G_{12}(\mathrm{GPa})$ & $v_{12}$ & $\varepsilon_{1}^{u}$ & $\varepsilon_{2}^{u}$ & $\gamma_{12}^{u}$ \\
\hline 127.59 & 13.03 & 6.41 & 0.31 & 0.008 & 0.029 & 0.015 \\
\hline
\end{tabular}

12 design variables to be selected from the three basic ply stacks of $0_{2}, 90_{2}$ and \pm 45 with no more than four contiguous plies of the same ply orientation. The optimization problem was solved by using the MCLACA. The parameters used in (5), (6), (7) and (8) and listed in Table 2 are determined based on the guideline presented in Haibin (2005) for the best performance.

It is noted that the optimisation solution is not unique in this problem. Similar to Kogiso et al. (1994), the optimal designs within $0.1 \%$ of the global optimum are accepted as an optimal solution called a practical optimum in this paper. In order to quantify the solution quality and computational efficiency of the MCLACA, an indicator called normalized price was introduced and used in this paper. It was used to assess and compare the MCLACA performance with the GA results in Kogiso et al. (1994). An additional indicator called practical reliability was also introduced to measure the probability of a solution reaching a practical optimum. For example, if 100 run optimization jobs are performed and 80 have reached the practical optimum, the practical reliability is 0.8 . The normalised price is defined as the average number of evaluations of the objective function divided by practical reliability. In this paper the average number of evaluations and practical reliability are obtained by performing 100 run of the MCLACA optimization for each of the three load cases. For comparison purpose, the best solutions and the corresponding results of $\lambda_{c b}$ and $\lambda_{c f}$ by using GA in Kogiso et al. (1994) are summarised in Table 3. Note that GA produces more than one optimum solution. Table 3 shows that six practical optimum designs were obtained for load case 1 , four optimum designs with two identical for load case 2 where the search process was difficult, and only one practical optimum design for load case 3 .

To study the effect of ant number on the MCLACA performance efficiency and practical reliability, different number of ants were selected and 100 runs was performed in the three load cases. Figure 5 shows the normalised price reduces with the selected ant number. The results show that the MCLACA performs very well in terms of computational

Table 2 Parameter values for the MCLACA

\begin{tabular}{lccccccc}
\hline Parameter & $\alpha$ & $q_{0}$ & $\xi$ & $\varepsilon$ & $\rho$ & $\tau_{0}$ & Number \\
\hline Value & 0.5 & 0.8 & 0.8 & 0.4 & 0.6 & 0.1 & 10 \\
\hline
\end{tabular}

Table 3 Selected practical optimal designs by GA (Kogiso et al. 1994)

\begin{tabular}{llrl}
\hline \multirow{2}{*}{$\begin{array}{l}\text { Load Stacking sequence } \\
\text { case }\end{array}$} & \multicolumn{2}{c}{ Loading factor } \\
\cline { 3 - 4 } & & \multicolumn{2}{c}{ Buckling $\lambda_{c b}$ Strain $\lambda_{c f}$} \\
\hline $1 \quad\left[ \pm 45_{5} / 0_{4} / \pm 45 / 0_{4} / 90_{2} / 0_{2}\right]_{\mathrm{s}}$ & $14,659.58$ & $13,518.66$ \\
& {$\left[ \pm 45_{5} / / 0_{4} / 90_{2} / 0_{4} / \pm 45 / 0_{2}\right]_{\mathrm{s}}$} & $14,610.85$ & $13,518.66$ \\
& {$\left[ \pm 45_{2} / 90_{2} / \pm 45 /\left( \pm 45 / 0_{4}\right)_{2} / \pm 45 / 0_{2}\right]_{\mathrm{s}}$} & $14,421.31$ & $13,518.66$ \\
& {$\left[ \pm 45_{4} / 0_{2} /\left( \pm 45 / 0_{4}\right)_{2} / 0_{4} / 90_{2}\right]_{\mathrm{s}}$} & $14,284.15$ & $13,518.66$ \\
& {$\left[ \pm 45_{4} / 0_{2} / \pm 45 / 0_{4} / 90_{2} / 0_{4} / \pm 45\right]_{\mathrm{s}}$} & $14,251.66$ & $13,518.66$ \\
& {$\left[ \pm 45_{2} / 90_{2} / 0_{2} / \pm 45_{2} / 0_{4} /\left( \pm 45 / 0_{2}\right)_{2}\right]_{\mathrm{s}}$} & $13,818.29$ & $13,518.66$ \\
& {$\left[ \pm 45_{2} / 90_{2} / \pm 45_{3} / 0_{2} / \pm 45 / 0_{4} / \pm 45 / 0_{2}\right]_{\mathrm{s}}$} & $12,743.45$ & $12,678.78$ \\
& {$\left[ \pm 45_{2} / 90_{2} / \pm 45_{4} /\left(0_{2} / \pm 45 / 0_{2}\right)_{2}\right]_{\mathrm{s}}$} & $12,725.26$ & $12,678.78$ \\
& {$\left[90_{2} / \pm 45_{5} /\left(0_{2} / \pm 45 / 0_{2}\right)_{2}\right]_{\mathrm{s}}$} & $12,674.85$ & $12,678.78$ \\
& {$\left[ \pm 45_{2} / 90_{2} / \pm 45_{3} / 0_{2} / \pm 45 / 0_{4} / \pm 45 / 0_{2}\right]_{\mathrm{s}}$} & $12,743.45$ & $12,678.78$ \\
3 & {$\left[90_{2} / \pm 45_{2} /\left(90_{2} / \pm 45\right)_{2} / \pm 45_{5}\right]_{\mathrm{s}}$} & $9,998.20$ & $10,398.14$ \\
& {$\left[90_{2} / \pm 45_{2} /\left(90_{2} / \pm 45\right)_{2} / \pm 45_{5}\right]_{\mathrm{s}}$} & $9,998.20$ & $10,398.14$ \\
\hline
\end{tabular}

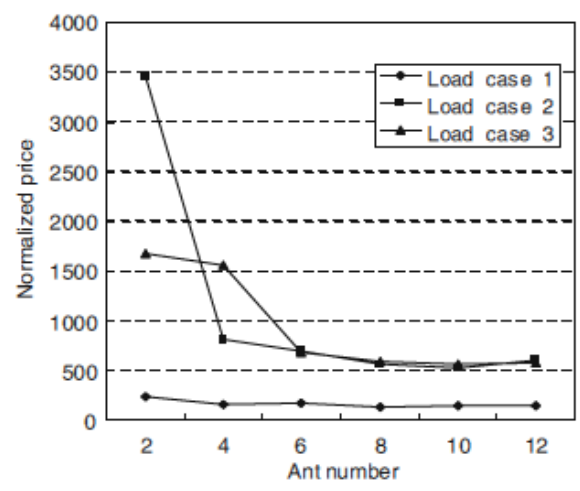

Fig. 5 Normalization price against ant number

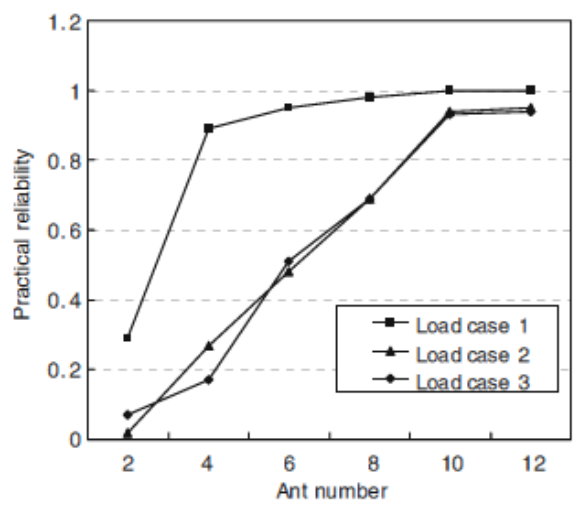

Fig. 6 Practical reliability against ant number 
Table 4 Practical optimal design of MCLACA

\begin{tabular}{llrl}
\hline \multirow{2}{*}{$\begin{array}{l}\text { Load Stacking sequence } \\
\text { case }\end{array}$} & \multicolumn{2}{l}{ Load factor } \\
\cline { 3 - 4 } & \multicolumn{2}{l}{ Buckling $\lambda_{c b}$ Strain $\lambda_{c f}$} \\
\hline 1 & {$\left[ \pm 45_{2} / 90_{2} / 0_{2} / \pm 45_{2} / 0_{4} /\left( \pm 45 / 0_{2}\right)_{2}\right]_{\mathrm{s}}$} & $13,818.29$ & $13,518.66$ \\
2 & {$\left[ \pm 45_{2} / 90_{2} / \pm 45_{3} / 0_{2} / \pm 45 / 0_{4} / \pm 45 / 0_{2}\right]_{\mathrm{s}}$} & $12,743.45$ & $12,678.78$ \\
3 & {$\left[90_{2} / \pm 45_{2} /\left(90_{2} / \pm 45\right)_{2} / \pm 45_{5}\right]_{\mathrm{s}}$} & $9,998.20$ & $10,398.14$ \\
\hline
\end{tabular}

cost for $\mathrm{m}>6$. Figure 6 shows the practical reliability increases with the ant number. It shows that the solution quality becomes high and stable when the ant number is beyond $\mathrm{m}=10$.

It is reported that the stop criterion, which was defined as the number of evaluations of objective function without improvement, has significant influence on the normalised

Table 5 Comparison of MCLACA and MCLACAWI with GA (Kogiso et al. 1994) and ACA (Aymerich and Serra 2008)

\begin{tabular}{|c|c|c|c|c|}
\hline $\begin{array}{l}\text { Load } \\
\text { case }\end{array}$ & $\begin{array}{l}\text { Stop } \\
\text { criterion }\end{array}$ & Algorithm & $\begin{array}{l}\text { Normalized } \\
\text { price }\end{array}$ & $\begin{array}{l}\text { Practical } \\
\text { reliability }\end{array}$ \\
\hline \multirow[t]{4}{*}{1} & \multirow[t]{4}{*}{133} & GA & 350 & 0.84 \\
\hline & & $\mathrm{ACA}$ & 201 & 0.99 \\
\hline & & MCLACAWI & 301 & 0.52 \\
\hline & & MCLACA & 148 & 1 \\
\hline \multirow[t]{4}{*}{2} & \multirow[t]{4}{*}{441} & GA & 1,250 & 0.78 \\
\hline & & $\mathrm{ACA}$ & 758 & 0.92 \\
\hline & & MCLACAWI & 1,179 & 0.53 \\
\hline & & MCLACA & 523 & 0.96 \\
\hline \multirow[t]{4}{*}{3} & \multirow[t]{4}{*}{266} & GA & 832 & 0.81 \\
\hline & & $\mathrm{ACA}$ & 686 & 0.71 \\
\hline & & MCLACAWI & 1,316 & 0.36 \\
\hline & & MCLACA & 536 & 0.83 \\
\hline \multirow[t]{4}{*}{1} & \multirow[t]{4}{*}{308} & GA & 530 & 0.99 \\
\hline & & $\mathrm{ACA}$ & 366 & 1 \\
\hline & & MCLACAWI & 294 & 0.85 \\
\hline & & MCLACA & 140 & 1 \\
\hline \multirow[t]{4}{*}{2} & \multirow[t]{4}{*}{308} & GA & 1,126 & 0.71 \\
\hline & & $\mathrm{ACA}$ & 631 & 0.84 \\
\hline & & MCLACAWI & 1,675 & 0.34 \\
\hline & & MCLACA & 334 & 0.95 \\
\hline \multirow[t]{4}{*}{3} & \multirow[t]{4}{*}{308} & GA & 963 & 0.77 \\
\hline & & $\mathrm{ACA}$ & 698 & 0.82 \\
\hline & & MCLACAWI & 1,381 & 0.50 \\
\hline & & MCLACA & 598 & 0.85 \\
\hline \multirow[t]{4}{*}{ Average } & \multirow[t]{4}{*}{308} & GA & 836 & 0.823 \\
\hline & & $\mathrm{ACA}$ & 489 & 0.887 \\
\hline & & MCLACAWI & 894 & 0.563 \\
\hline & & MCLACA & 345 & 0.933 \\
\hline
\end{tabular}

price (Aymerich and Serra 2008). For comparison purpose, the stop criterion used in the GA (Kogiso et al. 1994) and ACA (Aymerich and Serra 2008) was adapted in the MCLACA. In order to study the effect of the two point interchange operator, the MCLACA with and without the operator (MCLACAWI) by using the same parameters was employed in the three load cases. Table 4 shows the practical optimal solutions from MCLACA and the corresponding load factors $\lambda_{c b}$ and $\lambda_{c f}$. From Tables 3 and 4 , it is noted that the MCLACA can produce a practical optimum design which is identical to one of the GA results. Unlike the GA however, MCLACA produces a unique optimum solution. It is also noted that when the bi-axial load ratio $\mathrm{Ny} / \mathrm{Nx}$ is below 0.5 in case 1 and 2, the strain failure is slightly more critical than the buckling failure and the 0 -ply plays a certain role. When $\mathrm{Ny} / \mathrm{Nx}$ reaches 0.5 however, buckling becomes more critical and 0-ply has been excluded in the optimised laminate.

Table 5 presents the performance comparison between GA, ACA, MCLACA and MCLACAWI in terms of the normalised price and practical reliability under different stop criteria in the three load cases.

From Table 5, it can be observed that both the normalized price and practical reliability of the MCLACA without the two point interchange operator (MCLACAWI) are much worse in all load cases with different stop criterions. With the operator, MCLACA has an obvious improvement in

Table 6 Comparison of performance between MCLACA with GA (Kogiso et al. 1994) and ACA (Aymerich and Serra 2008 )

\begin{tabular}{llll}
\hline $\begin{array}{l}\text { Load } \\
\text { case }\end{array}$ & Algorithm & $\begin{array}{l}\text { Normalized } \\
\text { price }\end{array}$ & $\begin{array}{l}\text { Practical } \\
\text { reliability }\end{array}$ \\
\hline 1 & GA $_{1}$ & 580.8 & 0.99 \\
& GA $_{2}$ & 572.1 & 0.99 \\
& ACA & 463.4 & 1.0 \\
2 & MCLACA & 130.4 & 1.0 \\
& GA $_{1}$ & $1,302.9$ & 0.63 \\
& GA $_{2}$ & $1,119.3$ & 0.71 \\
& ACA & 736.7 & 0.85 \\
& MCLACA & 515.4 & 0.85 \\
& GA 1 & $1,109.2$ & 0.74 \\
& GA & 935.7 & 0.86 \\
& ACA & 908.6 & 0.81 \\
& MCLACA & 544.8 & 0.94 \\
Average & GA 1 & 940.1 & 0.79 \\
& GA & 846.3 & 0.85 \\
& ACA & 686.3 & 0.89 \\
& MCLACA & 387.3 & 0.93 \\
\hline
\end{tabular}

$\mathrm{GA}_{1}$ : population size $=8$; probability of permutation $=0.5 ; \mathrm{GA}_{2}$ : population size $=8 ;$ probability of permutation $=1.0$ 
Table 7 Optimised laminate layup and load factors in five boundary conditions

\begin{tabular}{llrr}
\hline \multirow{2}{*}{$\begin{array}{l}\text { Boundary } \\
\text { condition }\end{array}$} & Stacking sequence & \multicolumn{2}{l}{ Critical loading factor } \\
\cline { 3 - 4 } & & Buckling $\lambda_{c b}$ & Strain $\lambda_{c f}$ \\
\hline (S,F,F,S $)$ & {$\left[( \pm 45)_{12}\right]_{\mathrm{s}}$} & 160.77 & $25,760.07$ \\
$(\mathrm{~S}, \mathrm{~F}, \mathrm{~S}, \mathrm{~S})$ & {$\left[ \pm 60 / 90_{2} /( \pm 60)_{3} /\left(90_{2}\right)_{2} / \pm 60 /( \pm 45)_{4}\right]_{\mathrm{s}}$} & $5,798.86$ & $18,840.82$ \\
$(\mathrm{~S}, \mathrm{~S}, \mathrm{~S}, \mathrm{~S})$ & {$\left[\left( \pm 60 / 90_{2}\right)_{2} /( \pm 60)_{3} / 90_{2} / \pm 45 /\left(90_{2}\right)_{2} / \pm 60\right]_{\mathrm{s}}$} & $8,098.80$ & $13,935.32$ \\
$(\mathrm{C}, \mathrm{S}, \mathrm{S}, \mathrm{S})$ & {$\left[ \pm 60 /\left(90_{2}\right)_{2} /( \pm 60)_{2} / \pm 45 /\left(90_{2}\right)_{2} / \pm 60 / \pm 45 / \pm 60 / \pm 45\right]_{\mathrm{s}}$} & $15,734.08$ & $17,134.26$ \\
$(\mathrm{C}, \mathrm{S}, \mathrm{S}, \mathrm{C})$ & {$\left[ \pm 60 /\left(90_{2}\right)_{2} /( \pm 60)_{3} /\left(90_{2} / \pm 5\right)_{2} / \pm 45 / 90_{2}\right]_{\mathrm{s}}$} & $15,793.89$ & $17,045.30$ \\
\hline
\end{tabular}

practical reliability with a reduction in normalized price. When using a stop criterion of 308 in the example, an increase about 0.37 of the average practical reliability and a decrease at least $61 \%$ (894 vs 345) of the average normalized price were obtained by the MCLACA comparing with the MCLACAWI. The two-point interchange operator made the MCLACA performance better than the other three methods listed in Table 5.

Table 6 shows the performance results under a selected stopping criterion of 392 evaluations of the objective function without improvement in the three load cases. The results show that the MCLACA are better than the other two methods in terms of the normalized price and practical reliability. Compared with GA for example, a decrease of the average price over $70 \%$ (530 vs.140) with an increase of the average practical reliability has been achieved by MCLACA. Compared with ACA, a decrease of the average price over $60 \%$ ( $366 \mathrm{vs}$. 140$)$ with the same average practical reliability has been achieved by MCLACA. The results in Tables 5 and 6 also show that similar to GA and ACA the performance of the MCLACA search process is largely dependent on the load case.

To extend the application of the proposed MCLACA to a general case, an additional example of composite laminate optimisation was investigated. In this example, the 48-ply laminate dimension and materials remain the same as the benchmark case. The laminate layup is kept symmetric and balanced with ply contiguous constraints. Instead of the simple buckling formula however, MSC.NASTRAN with finite element method is employed to model the laminate and conduct the buckling analysis in the optimisation process. In this analysis, the stiffness coupling terms such as $D_{16}$ and $D_{26}$ and different boundary conditions has been taken into consideration. Five fibre orientation options $\{0$, $\pm 30, \pm 45, \pm 60,90\}$ are selected as design variables and five different boundary conditions are considered. Under the same applied load $\mathrm{Nx}=175 \mathrm{~N} / \mathrm{m}$ as the benchmark case and a larger load ratio of $\mathrm{Ny} / \mathrm{Nx}=0.8$, the optimised laminate layup and the corresponding critical load factors $\lambda_{c b}$ and $\lambda_{c f}$ in the five boundary conditions were obtained and listed in Table 7. In the table, (S,F,F,S), $(\mathrm{S}, \mathrm{F}, \mathrm{S}, \mathrm{S}),(\mathrm{S}, \mathrm{S}, \mathrm{S}, \mathrm{S}),(\mathrm{C}, \mathrm{S}, \mathrm{S}, \mathrm{S})$ and $(\mathrm{C}, \mathrm{S}, \mathrm{S}, \mathrm{C})$ represent the five boundary conditions in a combination of free(F), simply supported(S) and clamped(C) edges of the plate as illustrated in Fig. 1. For example, (S,F,F,S) represents a simply supported edge-1, free edge- 2 and 3 and simply supported edge-4.

The results in Table 7 show that the buckling load factor $\lambda_{c b}$ of the optimised plate increases significantly as more degrees of freedom in the boundary conditions are restricted. However a more restricted boundary condition may result in a smaller strain failure load although it is less critical in this case. As noted in the benchmark example, the buckling failure becomes more critical than the strain failure when the bi-axial load ratio $\mathrm{Ny} / \mathrm{Nx} \geq 0.5$. In addition, the 0 -ply has been excluded in the optimised laminate layup. Since a larger load ratio $\mathrm{Ny} / \mathrm{Nx}=0.8$ is taken in this current case, both $\lambda_{c b}$ and $\lambda_{c f}$ values in the (S,S,S,S) condition as shown in Table 7 are slightly smaller than the benchmark case 3 results shown in Table 4. It is also noted that instead of the dominating $75 \% \pm 45$ plies in the benchmark case, much less \pm 45 plies $(8.3 \%$ ) and more \pm 60 plies $(50 \%)$ and 90 plies $(42 \%)$ have been selected in this optimised laminate.

\section{Conclusions}

This paper introduced a new algorithm called the multi citylayer ant colony algorithm (MCLACA). It is developed to optimise the stacking sequence of a laminated plate for maximum buckling load subject to strain and ply contiguous constraints. The optimisation procedure is described as a travel salesman problem and solved by using the MCLACA.

Based on the benchmark case study of a laminate example and buckling analysis method used in previous research, it is noted that the performance of the MCLACA in terms of computational efficiency measured by the normalized price and the quality of solutions measured by the practical reliability is clearly better than the classical GA and ACA. Unlike the GA, the MCLACA produces a unique optimum solution. Under the specified ply orientation options and ply contiguous constraint, the MCLACA solution is practical and agrees with one of the GA results. When the MCLACA 
is applied to a general case of laminate optimisation with more design variables and five different boundary conditions, it also performs well together with the FEA used for laminate modelling and strain and buckling analysis. In both the benchmark and general case study, it is also noted that when the bi-axial load ratio $\mathrm{Ny} / \mathrm{Nx} \geq 0.5$ the laminate buckling failure becomes more critical than the strain failure.

\section{References}

Aymerich F, Serra M (2008) Optimization of laminate stacking sequence for maximum buckling load using the ant colony optimization(ACA) metaheuristic. Compos, Part A 39:262-272

Chen HP, Karunaratne R (2002) Optimum stacking sequence design of composite laminates using genetic algorithm. In: International SAMPE symposium and exhibition: proceedings, vol 47, pp 1402-1414

Coelho LDS, Mariani VC (2008) Use of chaotic sequences in a biologically inspired algorithm for engineering design optimization. Expert Syst App 34(3): 1905-1913

Dorigo M, Gambardella LM (1997) Ant colonies for the traveling salesman problem. BioSystems 43:73-81

Goldberg DE, Samtani MP (1986) Engineering optimization via genetic algorithm. In: Electronic computation, proceedings of the ninth conference, pp 471-482

Haibin D (2005) The theory and application of ant colony algorithm. Science Press, Beijing

Kameyama M, Fukunaga H (2007) Optimum design of composite plate wings for aeroelastic characteristics using lamination parameters. Comput Struct 85:213-224
Kogiso N, Watson LT, Guirdal Z, Haftka RT (1994) Genetic algorithms with local improvement for composite laminate design. Struct Optim 7:207-218

Kuan SN, Ong HL, Ng KM (2006) Solving the feeder bus network design problem by genetic algorithms and ant colony optimization. Adv Eng Softw 37(6):351-359

Le Riche R, Haftka RT (1993) Optimization of laminate stacking sequence for buckling load maximization by genetic algorithm. AIAA J 31(5):951-956

Le Riche R, Haftka RT (1995) Improved genetic algorithm for minimum thickness composite laminate design. Compos Eng $5(2): 143-161$

Muc A, Gurba W (2001) Genetic algorithms and finite element analysis in optimization of composite structures. Compos Struct $54(2-3): 275-281$

Park JH, Hwang JH, Lee CS, Hwang W (2001) Stacking sequence design of composite laminates for maximum strength using genetic algorithms. Compos Struct 52(2):217-231

Sciuva MD, Gherlone M, Lomario D (2003) Multiconstrained optimization of laminated and sandwich plates using evolutionary algorithms and higher-order plate theories. Compos Struct 59(1):149-154

Serra M, Venini P (2006) On some applications of ant colony optimization metaheuristic to plane truss optimization. Struct Multidisc Optim 32:499-506

Ugur A, Aydin D (2009) An interactive simulation and analysis software for solving TSP using ant colony optimization algorithms. Adv Eng Softw 40(5):341-349

Vinson JR, Sierakowski RL (1987) The behaviour of structures composed of composite materials. Kluwer, Dordrecht

Walker M, Smith RE (2003) A technique for the multiobjective optimization of laminated composite structures using genetic algorithms and finite element analysis. Compos Struct 62(1): $123-128$ 